\title{
Experimental evidence that phenotypic divergence in predators drives community divergence in prey
}

\author{
Eric P. Palkovacs ${ }^{1}$ and David M. Post \\ ${ }^{1}$ Department of Ecology and Evolutionary Biology, Yale University, New Haven, Connecticut 06520-8106 USA
}

\begin{abstract}
Studies of adaptive divergence have traditionally focused on the ecological causes of trait diversification, while the ecological consequences of phenotypic divergence remain relatively unexplored. Divergence in predator foraging traits, in particular, has the potential to impact the structure and dynamics of ecological communities. To examine the effects of predator trait divergence on prey communities, we exposed zooplankton communities in lake mesocosms to predation from either anadromous or landlocked (freshwater resident) alewives, which have undergone recent and rapid phenotypic differentiation in foraging traits (gape width, gill raker spacing, and prey size-selectivity). Anadromous alewives, which exploit large prey items, significantly reduced the mean body size, total biomass, species richness, and diversity of crustacean zooplankton relative to landlocked alewives, which exploit smaller prey. The zooplankton responses observed in this experiment are consistent with patterns observed in lakes. This study provides direct evidence that phenotypic divergence in predators, even in its early stages, can play a critical role in determining prey community structure.
\end{abstract}

Key words: adaptive divergence; alewife; Alosa pseudoharengus; eco-evolutionary interactions; extended phenotype; gill rakers; intraspecific variation; niche construction; phenotypic divergence; predator-prey interactions; size-selective predation; zooplankton.

\section{INTRODUCTION}

Ecological diversification in vertebrates commonly involves divergence in traits related to prey capture and/or consumption (Schluter 2000). Examples include the well-known radiations of Darwin's finches (Lack 1947, Grant 1999), threespine sticklebacks (Bell and Foster 1994, McKinnon and Rundle 2002), and African cichlids (Kocher 2004, Seehausen 2006). Because differences in prey choice can drive the initial stages of phenotypic divergence and begin the process of ecological speciation, much is known about the ecological causes of diversification in foraging traits (see Dieckmann et al. 2004). Considerably less is known about the ecological consequences of phenotypic diversification in predator foraging traits. It is well established that predators can have strong influences on prey community structure (Brooks and Dodson 1965, Paine 1966, Carpenter et al. 1985, Sih et al. 1985), and that different predator species generate different prey communities as a result of differences in morphology and foraging behavior (McPeek 1998, Schmitz and Suttle 2001, Chalcraft and Resetarits 2003, Straub and Snyder 2006). But what are the ecological consequences of phenotypic divergence in its early stages?

Manuscript received 8 September 2008; accepted 22 September 2008. Corresponding Editor: D. E. Schindler.

${ }^{1}$ Present address: School of Biology and Ecology, University of Maine, Orono, Maine 04469-5751 USA.

E-mail: eric.palkovacs@maine.edu
Here we experimentally evaluated the hypothesis that phenotypic differences among populations of a predator drive differences in the structure of the prey community. To test this hypothesis, we exposed zooplankton communities in lake mesocosms to predation by either anadromous or landlocked (freshwater resident) forms of a dominant planktivorous fish, the alewife (Alosa pseudoharengus). The seminal work of Brooks and Dodson (1965) on landlocked alewife-zooplankton interactions was fundamental in establishing the ability of predators to structure prey communities. However, differences in morphology and prey selectivity suggest that the ecological impacts of anadromous alewives may differ from those of landlocked alewives.

Landlocked alewife populations in Connecticut (USA) have diverged in foraging traits from the anadromous populations that founded them. This phenotypic divergence has led landlocked populations to display narrower gape width and smaller gill raker spacing than anadromous populations (Palkovacs and Post 2008, Palkovacs et al. 2008). In alewives, as in other planktivorous fishes, there exists a positive correlation between gill raker spacing and optimal prey size (MacNeill and Brandt 1990). Anadromous alewives, with their larger gill raker spacings, show a stronger preference for large prey items compared to landlocked alewives (Palkovacs and Post 2008). Differences in prey availability between landlocked alewife lakes and anadromous alewife lakes (and the marine environment) suggest that phenotypic differences are adaptive (Palko- 
vacs and Post 2008), and genetic data show that phenotypic differences have emerged rapidly (in the past 300-5000 years) and repeatedly in independently isolated landlocked populations (Palkovacs et al. 2008).

Data from Connecticut lakes suggest that differences in alewife traits may have significant impacts on zooplankton prey communities (Post et al. 2008). Landlocked alewives are present in freshwater lakes year-round, placing constant predation pressure on zooplankton communities (Brooks and Dodson 1965, Post et al. 2008). In contrast, anadromous alewives are present in freshwater between March, when spawning begins, and November, when the annual out-migration of juveniles to the sea is complete (Kissil 1974, Loesch 1987). Anadromous alewives are iteroparous, and adults usually return to the sea after several days or weeks in freshwater. Therefore, the biomass of juveniles in lakes, and the predation pressure exerted by them, far exceeds that of adults throughout most of the growing season (D. M. Post and E. P. Palkovacs, unpublished data). The out-migration of anadromous juveniles creates a temporal refuge for zooplankton that allows zooplankton body size and biomass to rebound annually. However, the annual spring onset of anadromous alewife predation induces rapid declines in zooplankton body size and biomass, causing anadromous alewives to have stronger, more dynamic seasonal effects on freshwater zooplankton communities than do landlocked alewives (Post et al. 2008).

Two hypotheses could explain the apparent stronger effects of anadromous alewives on natural zooplankton communities. First, differences in community effects could result from phenotypic divergence in predator foraging traits, including gape width, gill raker spacing, and prey selectivity. Alternatively, differences in community effects could result from ecological factors, including differences in alewife abundance or differences in body size due to ontogeny.

Data from lakes suggest that phenotypic divergence in alewife foraging traits drives differences in zooplankton size, biomass, and community structure (Palkovacs and Post 2008, Post et al. 2008). The preference for largebodied prey shown by anadromous alewives may eliminate large-bodied zooplankton from the environment, whereas the ability of landlocked alewives to exploit small prey may allow some large-bodied zooplankton to persist. However, this interpretation is based on field observations. While observational studies can show consistency with a given hypothesis, they cannot conclusively rule out alternatives; for this, an experimental approach is needed.

Here we used experimental mesocosms to test the effects of alewife trait divergence on the biomass, mean body size, diversity, species richness, and structure of the zooplankton prey community. We began with identical zooplankton communities, controlled for ecological factors including alewife density and body size, and tested whether phenotypic divergence produced zoo- plankton community differences consistent with those observed in lakes.

\section{Methods \\ Experimental procedure}

Twelve clear, solid-walled polyethylene mesocosms (2 $\mathrm{m}$ diameter $\times 6 \mathrm{~m}$ deep) were suspended in Rogers Lake (Old Lyme, Connecticut, USA) on 8 June 2004. Each mesocosm was filled with unfiltered lake water so that all began with ambient zooplankton communities. The mesocosms were left unmanipulated for three weeks to enable the establishment of zooplankton communities. Treatments (anadromous, landlocked, or no fish) were randomly assigned and, on 24 June, mesocosms were stocked with either 15 landlocked or 15 anadromous young-of-the-year (YOY) or left unstocked (no fish). This stocking rate $\left(4.8 \mathrm{fish} / \mathrm{m}^{2}\right)$ is within the range of densities of anadromous alewife YOY found in Connecticut lakes during early to mid-June (Palkovacs and Post 2008). Landlocked and anadromous alewives for stocking were captured from Rogers Lake and Gorton Pond (East Lyme, Connecticut, USA), respectively, using a purse seine (depth $4.9 \mathrm{~m}$, which encloses an area of $100 \mathrm{~m}^{2}$ ) and stocked directly into the mesocosms. Because alewives for this experiment were wild-caught from different lakes, the community responses being measured are net phenotypic effects (the sum of genetic effects plus plastic effects).

Stocked fish were between 30 and $45 \mathrm{~mm}$ total length, and mean length at stocking did not differ significantly between the groups. At the termination of the experiment, all fish were removed from the mesocosms and frozen for preservation. For each specimen, total length, mass, gill raker spacing, and gape width were measured according to Palkovacs et al. (2008). Values for alewife density and size did not differ significantly between anadromous and landlocked treatments at the termination of the experiment: alewife density (anadromous, 6.0 $\pm 2.48 \mathrm{fish} /$ mesocosm, mean $\pm \mathrm{SE}$; landlocked, $6.5 \pm$ $1.04 \mathrm{fish} / \mathrm{mesocosm}$ ), alewife mass (anadromous, $0.99 \pm$ $0.09 \mathrm{~g}$; landlocked, $1.03 \pm 0.07 \mathrm{~g})$, total length (anadromous, $51.21 \pm 1.05 \mathrm{~mm}$; landlocked, $51.69 \pm$ $1.23 \mathrm{~mm}$ ), condition factor (mass/total length ${ }^{3}$ ) (anadromous, $7.01 \times 10^{-6} \pm 3.02 \times 10^{-7}$, landlocked, $7.11 \times$ $\left.10^{-6} \pm 9.49 \times 10^{-8}\right)$.

Response variables of interest included the mean length of crustacean zooplankton, the biomass of crustacean zooplankton, total crustacean species richness, and Shannon diversity (calculated from relative biomass data). To quantify these variables, zooplankton communities in mesocosms were sampled weekly from 22 June to 3 August. Sampling was performed by towing an $80-\mu \mathrm{m}$ mesh plankton net with a $30 \mathrm{~cm}$ diameter mouth vertically from a depth of $5 \mathrm{~m}$. Zooplankton samples were preserved in $70 \%$ ethanol. Zooplankton were identified and enumerated under a dissecting microscope. When the numbers of organisms were large ( $>1000$ individuals), samples were split using a Folsom 

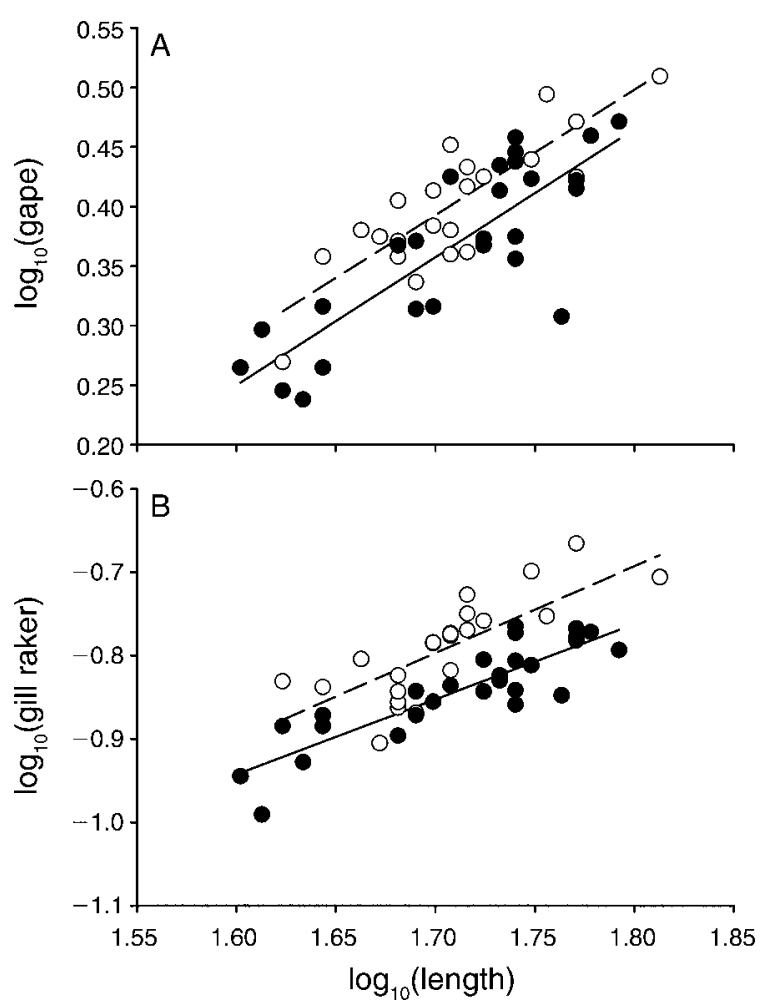

FIG. 1. Log-transformed (A) gape width and (B) gill raker spacing plotted against log-transformed total length for anadromous alewives (open circles, dashed regression line) and landlocked alewives (solid circles, solid regression line) from the experimental mesocosms.

plankton splitter. The first 100 crustaceans were measured and organisms were identified to species. For analysis, immature copepods were grouped together, and Diaptomus and Diaphanosoma species were grouped at the genus level. Zooplankton measurements and biomass estimations were performed according to Post et al. (2008). Rotifers were excluded from our analysis because they rarely occur in alewife diets (Palkovacs 2007) and underwent large magnitude cycles in the mesocosms that obscured biomass patterns in the primary crustacean prey community. Also excluded from analysis were copepod nauplii and littoral colonists (Acroperus, Alona, Alonella, Camptocercus, Eurycercus, Graptolebris, and Picripleuroxus), which appeared in the mesocosms at low abundances near the end of the experiment (maximum abundance, 5.9\%; mean abundance, $0.7 \%$ ) but are not representative of the typical pelagic alewife prey community. Response variables were also calculated for natural zooplankton communities from anadromous and landlocked lakes sampled from 2004 to 2006 (sampling details provided in Post et al. 2008).

\section{Statistical analysis}

To determine whether gape width and gill raker spacing differed between anadromous and landlocked alewives removed from the experiment, we applied ANCOVA, with alewife total length as a covariate. All morphological variables were $\log _{10}$-transformed for analysis. We used ANOVA to test whether zooplankton species richness, diversity, biomass, and mean body size differed between treatments before fish were added. Because we expected autocorrelation from one week to the next within individual mesocosms, we applied repeated-measures ANOVA to test for differences in response variables between anadromous and landlocked treatments (29 June-3 August). Biomass was $\log _{10^{-}}$ transformed to normalize data, and parametric statistics were performed using SPSS 11.0.4 (SPSS 2005).

To examine changes in zooplankton community structure over the course of the experiment, we used analysis of similarity (ANOSIM), which utilizes a nonparametric permutation/randomization procedure to test for significant differences between treatments based on a similarity matrix (Clarke 1993). We based our analysis on untransformed Euclidean distances calculated from relative biomass data. We tested each week of the experiment independently and ran 999 permutations per test. We also calculated similarity percentages (SIMPER) to identify which species contributed to similarity within treatments and dissimilarity between treatments (up to 90\%). ANOSIM and SIMPER were performed using the software package PRIMER 5 (Primer-E, Ivybridge, UK).

\section{RESUlts}

Slopes for $\log _{10}$-transformed morphological traits regressed on $\log _{10}$-transformed alewife total length did not differ for either gape width $\left(F_{1,46}=0.010, P=0.921\right)$ or gill raker spacing $\left(F_{1,46}=0.450, P=0.506\right)$. Because slopes were not significantly different, the interaction term was removed from the model and mean differences were evaluated. Anadromous alewives were found to have significantly wider gapes $\left(F_{1,47}=12.104, P=0.001\right)$ and larger gill raker spacings $\left(F_{1,47}=35.007, P<0.001\right)$ than landlocked alewives (Fig. 1), supporting the pattern of phenotypic divergence observed in lakes.

Tests of initial conditions confirmed that on 22 June, before fish were added, treatments did not differ in zooplankton biomass (ANOVA: $F_{1,6}=0.239, P=0.642$ ), mean zooplankton body length $\left(F_{1,6}=0.186, P=0.681\right)$, species richness $\left(F_{1,6}=0.429, P=0.537\right)$, or diversity $\left(F_{1,6}=0.134, P=0.727\right)$. After fish were added, the anadromous treatment developed significantly lower biomass $\left(F_{1,6}=6.389, P=0.045\right)$, mean body size $\left(F_{1,6}\right.$ $=7.061, P=0.038)$, species richness $\left(F_{1,6}=7.244, P=\right.$ $0.036)$, and diversity $\left(F_{1,6}=8.171, P=0.029\right)$, than the landlocked treatment (Fig. 2). The time $\times$ treatment interaction was not significant for any response variable, indicating that the slopes of the responses over time did not differ between anadromous and landlocked treatments. Therefore, the mean differences between treatments emerged during the first week of the experiment and were maintained over its duration. 

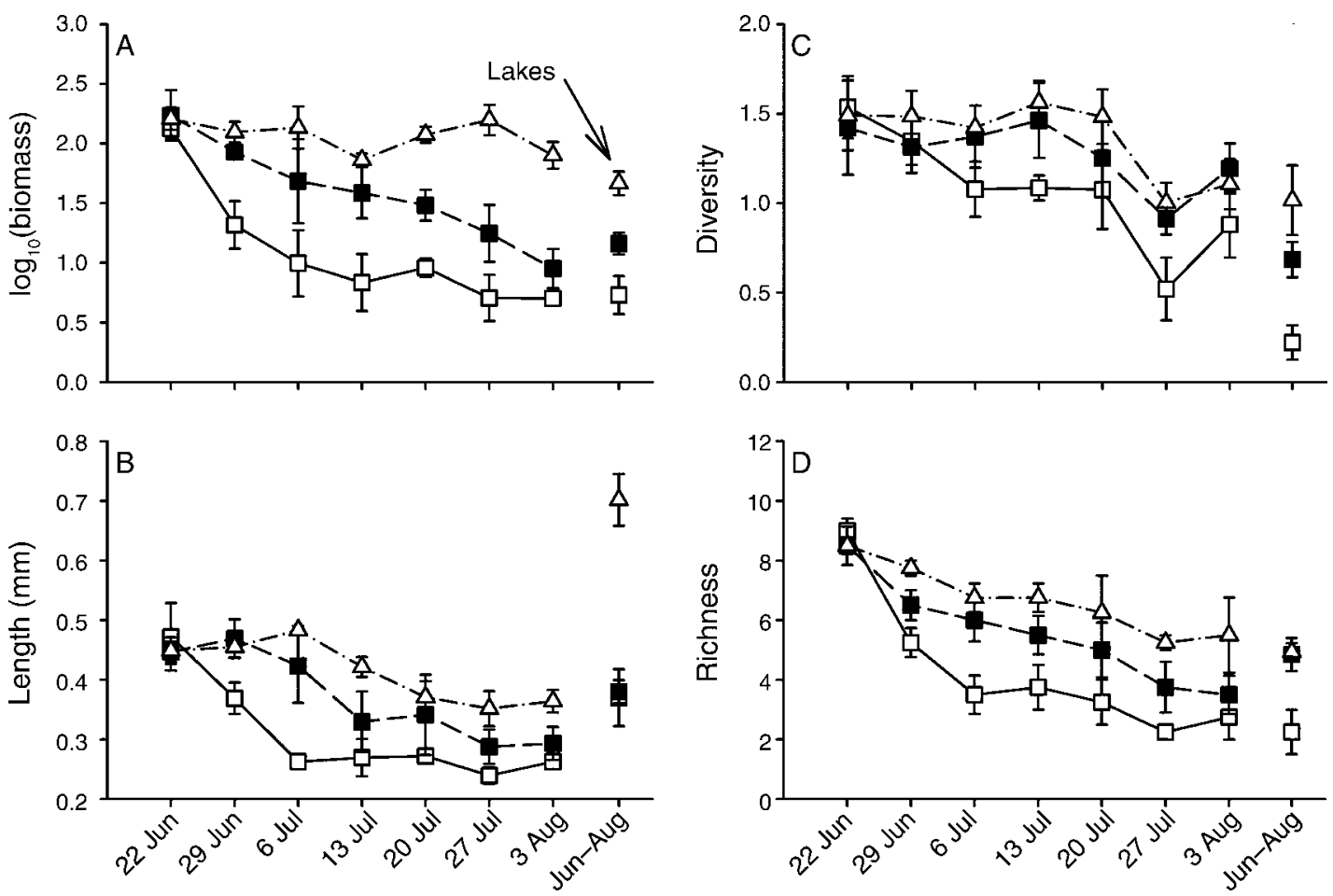

FIG. 2. (A) Log-transformed biomass, (B) body length, (C) Shannon diversity, and (D) species richness of crustacean zooplankton measured weekly throughout the duration of the experiment (mean $\pm \mathrm{SE}$ ) for treatments containing anadromous alewives (open squares), landlocked alewives (solid squares), or no alewives (open triangles). Fish were added on 24 June. The right side of each panel shows June-August values from lakes sampled from 2004 to 2006 (anadromous, $n=3$ lakes; landlocked, $n=8$; no alewife, $n=8)$.

As with other response variables, we detected no differences in zooplankton community structure between landlocked and anadromous treatments before fish were added (ANOSIM: $R=-0.198, P=0.999$ ). A significant difference in community structure between the anadromous and landlocked treatments occurred the second week after fish were introduced to the mesocosms $(R=0.656, P=0.029$; Table 1$)$. Largebodied species (mean length $>0.5 \mathrm{~mm}$ ), including Diaptomus spp. (contributing 5\% to community dissimilarity) and Holopeduim gibberum $(<5 \%)$ were com- pletely eliminated from the anadromous alewife mesocosms but persisted in the landlocked alewife mesocosms. Small-bodied species (mean length $<0.4$ $\mathrm{mm}$ ), including immature copepods (contributing $16 \%$ to community dissimilarity), Bosmina longirostris (22\%), and Chydorus sphaericus (11\%), dominated the anadromous alewife mesocosms. In contrast, medium-bodied species (mean length between 0.4 and $0.5 \mathrm{~mm}$ ), including Ceriodaphnia lacustris (contributing $12 \%$ to community dissimilarity) and Diaphanosoma spp. (26\%), dominated the landlocked alewife mesocosms.

TABLE 1. Average body length and biomass for the primary species contributing to the dissimilarity between zooplankton communities measured on the second week after fish were added to the experiment.

\begin{tabular}{llcccc}
\hline \hline & & & \multicolumn{3}{c}{ Biomass $(\mu \mathrm{g} / \mathrm{L})$} \\
\cline { 4 - 6 } \multicolumn{1}{c}{ Species } & Group & Length $(\mathrm{mm})$ & Anadromous & Landlocked & No fish \\
\hline Diaptomus spp. & copepod & 0.93 & $0.00(0)$ & $8.06(7)$ & $12.89(8)$ \\
Holopedium gibberum & cladoceran & 0.59 & $0.00(0)$ & $0.75(1)$ & $11.44(7)$ \\
Diaphanosoma spp. & cladoceran & 0.48 & $4.56(24)$ & $68.60(61)$ & $58.82(35)$ \\
Ceriodaphnia lacustris & cladoceran & 0.40 & $0.49(3)$ & $17.60(16)$ & $61.31(36)$ \\
Immature copepods & copepod & 0.28 & $1.41(7)$ & $1.28(1)$ & $5.38(3)$ \\
Bosmina longirostris & cladoceran & 0.27 & $7.50(39)$ & $7.57(7)$ & $9.02(5)$ \\
Chydorus sphaericus & cladoceran & 0.22 & $3.48(18)$ & $2.05(2)$ & $3.58(2)$ \\
Total biomass & & & 19.30 & 112.41 & 169.45 \\
& & & &
\end{tabular}

Notes: Total biomass represents the mean for all crustaceans (including species not represented in the table) for each treatment. The percentage contribution of each species to the total biomass for each treatment is provided in parentheses. 
Differences in community structure were not significant in other weeks.

\section{DisCUSSION}

Our results show that phenotypic divergence in foraging traits between two distinct alewife populations, one anadromous and one landlocked, has important consequences for zooplankton prey communities. Anadromous and landlocked alewives collected from the mesocosms at the end of our experiment differed significantly in morphological traits related to prey capture and retention. Anadromous alewives had significantly wider gapes and larger gill raker spacings than did landlocked alewives (Fig. 1). These morphological patterns are consistent with those observed in wild populations, where anadromous alewives also show wider gapes and larger gill raker spacings than landlocked alewives and consistently select larger zooplankton prey (Palkovacs and Post 2008, Palkovacs et al. 2008).

In our experimental mesocosms, phenotypic divergence in alewife foraging traits led to divergence in zooplankton prey communities (Table 1, Fig. 2). Predation by size-selective anadromous alewives eliminated large-bodied zooplankton species (Diaptomus spp. and Holopedium gibberum) and reduced the biomass of medium-bodied species (Diaphanosoma spp., and Ceriodaphnia lacustris), causing zooplankton communities to become dominated by small-bodied cladocerans (Bosmina longirostris and Chydorus sphaericus) and immature copepods. In contrast, zooplankton communities exposed to predation by landlocked alewives, which are not as size selective, retained large-bodied species and became dominated by medium-bodied species (Diaphanosoma spp. and Ceriodaphnia lacustris). By selectively preying on the largest zooplankton in the environment, anadromous alewives significantly reduced the total biomass, average body size, diversity, and species richness of their prey community relative to landlocked alewives (Table 1, Fig. 2).

The differences we observed in the mesocosms are consistent with those seen in zooplankton communities naturally co-occurring with either anadromous or landlocked alewife populations (Fig. 2). Midsummer zooplankton communities in anadromous alewife lakes are dominated by small-bodied Bosmina longirostris and immature copepods (Post et al. 2008), whereas zooplankton communities in landlocked alewife lakes are composed of medium-bodied Ceriodaphnia lacustris and Tropocyclops extensus (prasinus), small-bodied Bosmina longirostris, and immature copepods (Brooks and Dodson 1965, Post et al. 2008). In all experimental treatments we found a higher proportion of Diaphanosoma spp. and a lower proportion of mature cyclopoid copepods in the mesocosms than occur in lakes, likely reflecting species-specific responses to mesocosm conditions. Treatment effects emerged in the first few weeks of the experiment and then some convergence of treatments occurred as the experiment progressed. This convergence likely resulted from experimental artifacts, most notably the extinction of large-bodied species, even in the absence of fish (Fig. 2). For example, large cyclopoid copepods such as Mesocyclops edax, a primary prey item for anadromous alewives in lakes (Palkovacs 2007) did not persist in any of the mesocosms.

Evidence from morphologically divergent populations of several fish species suggests that differences in gill raker morphology have a large genetic component (Hagen 1973, Foote et al. 1999, Funk et al. 2005). However, phenotypic plasticity is also known to shape important aspects of foraging morphology in fishes (Meyer 1987, Mittelbach et al. 1999). Because we utilized wild-caught alewives, our experimental design is unable to parse genetic effects from plastic effects. Separating these effects is of interest for questions involving the eco-evolutionary dynamics of the alewifezooplankton system. If genetic effects are large, as has been found for a variety of plant-arthropod systems (reviewed in Whitham et al. 2006), then changes in zooplankton communities brought on by alewife predation potentially feed back to influence the trajectory of alewife evolution (Palkovacs and Post 2008).

\section{CONCLUSIONS}

The diversification of foraging traits is an important feature of many adaptive radiations and, thus, underlies much of the world's animal diversity (Schluter 2000). While the ecological causes of phenotypic divergence in predator foraging traits have been investigated widely, the ecological consequences of foraging trait divergence remain relatively unknown (but see Yonekura et al. 2007). Data from lakes suggest that alewife trait divergence drives ecological differences in zooplankton communities (Post et al. 2008). However, the effects of ecological factors, such as predator density and body size, are difficult to separate from the effects of evolutionary trait divergence based on observational data alone. By utilizing an experimental approach, we were able to parse the effects of trait divergence from those of ecological factors including density and body size. Our results show that phenotypic divergence in alewife foraging traits drove significant divergence in zooplankton prey communities.

In the context of understanding the process of phenotypic divergence, it is important to consider how ecological differences between prey communities, brought on by subtle differentiation in predator traits, might feed back to further influence trait divergence. Feedbacks may either facilitate divergence by driving phenotypes further apart or prevent divergence by pulling phenotypes back together. These types of reciprocal interactions between phenotypes and environments have been considered in theoretical models (Laland et al. 1999, Loeuille et al. 2002) and simple model ecosystems (Bohannan and Lenski 2000, Yoshida et al. 2003, Habets et al. 2006), but have yet to be 
examined in detail for complex natural ecosystems. Our findings suggest that a closer examination of such feedbacks could reveal much about the processes shaping predator phenotypes, mediating species interactions, and regulating prey communities.

\section{ACKNOWLEDGMENTS}

We thank C. Winkworth, B. Kochin, L. Puth, and C. Burns for help in the field and R. Paine, S. Stearns, D. Skelly, A. Hendry, C. Burns, and three anonymous reviewers for comments that greatly improved this manuscript. This research was supported by an EPA STAR Fellowship to E. P. Palkovacs, the Quebec-Labrador Foundation, the Connecticut Institute for Water Resources, and the National Science Foundation (DEB no. 0717265). All handling of fish was approved by the Yale University IACUC (Protocol no. 200310734) and the Connecticut DEP (Scientific Collector Permit no. SC-04016).

\section{Literature Cited}

Bell, M. A., and S. A. Foster, editors. 1994. The evolutionary biology of the threespine stickleback. Oxford University Press, Oxford, UK.

Bohannan, B. J. M., and R. E. Lenski. 2000. Linking genetic change to community evolution: insights from studies of bacteria and bacteriophage. Ecology Letters 3:362-377.

Brooks, J. L., and S. I. Dodson. 1965. Predation, body size, and composition of plankton. Science 150:28-35.

Carpenter, S. R., J. F. Kitchell, and J. R. Hodgson. 1985. Cascading trophic interactions and lake productivity. BioScience 35:634-639.

Chalcraft, D. R., and W. J. J. Resetarits. 2003. Predator identity and ecological impacts: functional redundancy or functional diversity? Ecology 84:2407-2418.

Clarke, K. R. 1993. Non-parametric multivariate analyses of changes in community structure. Australian Journal of Ecology 18:117-143.

Dieckmann, U., M. Doebeli, J. A. J. Metz, and D. Tautz, editors. 2004. Adaptive speciation. Cambridge University Press, Cambridge, UK.

Foote, C. J., K. Moore, K. Stenberg, K. J. Craig, J. K. Wenburg, and C. C. Wood. 1999. Genetic differentiation in gill raker number and length in sympatric anadromous and nonanadromous morphs of sockeye salmon, Oncorhynchus nerka. Environmental Biology of Fishes 54:263-274.

Funk, W. C., J. A. Tyburczy, K. L. Knudsen, K. R. Lindner, and F. W. Allendorf. 2005. Genetic basis of variation in morphological and life-history traits of a wild population of pink salmon. Journal of Heredity 96:24-31.

Grant, P. R. 1999. Ecology and evolution of Darwin's finches. Princeton University Press, Princeton, New Jersey, USA.

Habets, M. G. J. L., D. E. Rozen, R. F. Hoekstra, and J. A. G. M. de Visser. 2006. The effect of population structure on the adaptive radiation of microbial populations evolving in spatially structured environments. Ecology Letters 9:10411048.

Hagen, D. W. 1973. Inheritance of numbers of lateral plates and gillrakers in Gasterosteus aculeatus. Heredity 30:273-287.

Kissil, G. W. 1974. Spawning of anadromous alewife, Alosa pseudoharengus, in Bride Lake, Connecticut. Transactions of the American Fisheries Society 103:312-317.

Kocher, T. D. 2004. Adaptive evolution and explosive speciation: the cichlid fish model. Nature Reviews Genetics 5:288-298.

Lack, D. 1947. Darwin's finches. Cambridge University Press, Cambridge, UK.

Laland, K. N., F. J. Odling-Smee, and M. W. Feldman. 1999. Evolutionary consequences of niche construction and their implications for ecology. Proceedings of the National Academy of Sciences (USA) 96:10242-10247.

Loesch, J. G. 1987. Overview of life history aspects of anadromous alewife and blueback herring in freshwater habitats. American Fisheries Society Symposium 1:89-103.

Loeuille, N., M. Loreau, and R. Ferrière. 2002. Consequences of plant-herbivore coevolution on the dynamics and functioning of ecosystems. Journal of Theoretical Biology 217:369-381.

MacNeill, D. B., and S. B. Brandt. 1990. Ontogenetic shifts in gill-raker morphology and predicted prey capture efficiency of the alewife, Alosa pseudoharengus. Copeia 1990:164-171.

McKinnon, J. S., and H. D. Rundle. 2002. Speciation in nature: the threespine stickleback model systems. Trends in Ecology and Evolution 17:480-488.

McPeek, M. A. 1998. The consequences of changing the top predator in a food web: a comparative experimental approach. Ecological Monographs 68:1-23.

Meyer, A. 1987. Phenotypic plasticity and heterochrony in Cichlasoma managuense (Pisces, Cichlidae) and their implications for speciation in cichlid fishes. Evolution 41:1357-1369.

Mittelbach, G. C., C. W. Osenberg, and P. C. Wainwright. 1999. Variation in feeding morphology between pumpkinseed populations: phenotypic plasticity or evolution? Evolutionary Ecology Research 1:111-128.

Paine, R. T. 1966. Food web complexity and species diversity. American Naturalist 100:65-75.

Palkovacs, E. P. 2007. Feedbacks between ecology and evolution: linking the causes and consequences of functional biodiversity. Dissertation. Yale University, New Haven, Connecticut, USA.

Palkovacs, E. P., K. B. Dion, D. M. Post, and A. Caccone. 2008. Independent evolutionary origins of landlocked alewife populations and rapid parallel evolution of phenotypic traits. Molecular Ecology 17:582-597.

Palkovacs, E. P., and D. M. Post. 2008. Eco-evolutionary interactions between predators and prey: can predatorinduced changes to prey communities feed back to shape predator foraging traits. Evolutionary Ecology Research 10: 699-720.

Post, D. M., E. P. Palkovacs, E. G. Schielke, and S. I. Dodson. 2008. Intraspecific phenotypic variation in a predator affects community structure and cacading trophic interactions. Ecology 89:2019-2032.

Schluter, D. 2000. The ecology of adaptive radiation. Oxford University Press, Oxford, UK.

Schmitz, O. J., and K. B. Suttle. 2001. Effects of a top predator species on direct and indirect interactions in a food web. Ecology 82:2072-2081.

Seehausen, O. 2006. African cichlid fish: a model system in adaptive radiation research. Proceedings of the Royal Society B 273:1987-1998.

Sih, A., P. Crowley, M. A. McPeek, J. Petranka, and K. Strohmeier. 1985. Predation, competition, and prey communities: a review of field experiments. Annual Review of Ecology and Systematics 16:269-311.

SPSS. 2005. SPSS version 11.0.4 for Mac OS X. SPSS, Chicago, Illinois, USA.

Straub, C. S., and W. E. Snyder. 2006. Species identity dominates the relationship between predator biodiversity and herbivore suppression. Ecology 87:277-282.

Whitham, T. G., et al. 2006. A framework for community and ecosystem genetics: from genes to ecosystems. Nature Reviews Genetics 7:510-523.

Yonekura, R., Y. Kohmatsu, and M. Yuma. 2007. Difference in the predation impact enhanced by morphological divergence between introduced fish populations. Biological Journal of the Linnean Society 91:601-610.

Yoshida, T., L. E. Jones, S. P. Ellner, G. F. Fussmann, and N. G. J. Hairston. 2003. Rapid evolution drives ecological dynamics in a predator-prey system. Nature 424:303-306. 